\title{
Effects of a single session of SMR neurofeedback training on anxiety and cortisol levels
}

\author{
Marien Gadea ${ }^{a, *}$, Marta Aliñob ${ }^{b}$ Vanesa Hidalgo ${ }^{c}$, Raul Espert $^{a}$, \\ Alicia Salvador ${ }^{d}$
}

a Department of Psychobiology, University of Valencia, Valencia, Spain

b Department of Psychology, Universidad Internacional de Valencia, Valencia, Spain

c Area of Psychobiology, Department of Psychology and Sociology, University of Zaragoza, Zaragoza, Spain

${ }^{d}$ Laboratory of Cognitive Social Neuroscience, Department of Psychobiology and IDOCAL, University of

Valencia, Valencia, Spain

Received 10 December 2019; accepted 18 March 2020

Available online 9 April 2020

\section{KEYWORDS \\ Anxiety; \\ Cortisol; \\ Neurofeedback; \\ Sham procedure; \\ SMR rhythm}

\begin{abstract}
Summary
Objectives. - According to some studies, a putatively calming effect of EEG neurofeedback training could be useful as a therapeutic tool in psychiatric practice. With the aim of elucidating this possibility, we tested the efficacy of a single session of $\uparrow$ sensorimotor (SMR)/ $\downarrow$ theta neurofeedback training for mood improvement in 32 healthy men, taking into account trainability, independence and interpretability of the results.

Methods. - A pre-post design, with the following dependent variables, was applied: (i) psychometric measures of mood with regards to anxiety, depression, and anger (Profile of Mood State, POMS, and State Trait Anxiety Inventory, STAI); (ii) biological measures (salivary levels of cortisol); (iii) neurophysiological measures (EEG frequency band power analysis). In accordance with general recommendations for research in neurofeedback, a control group receiving sham neurofeedback was included.

Results. - Anxiety levels decreased after the real neurofeedback and increased after the sham neurofeedback $(P<0.01$, size effect 0.9 for comparison between groups). Cortisol decreased after the experiment in both groups, though with significantly more pronounced effects in the desired direction after the real neurofeedback $(P<0.04$; size effect 0.7$)$. The group receiving real neurofeedback significantly enhanced their SMR band $(P<0.004$; size effect 0.88$)$, without changes in the theta band. The group receiving sham neurofeedback did not show any EEG changes.
\end{abstract}

* Corresponding author. Departament de Psicobiologia, Facultat de Psicologia, Universitat de València, Avda. Blasco Ibañez 21, 46010 València, Spain.

E-mail address: Marien.Gadea@uv.es (M. Gadea). 
Conclusions. - The improvement observed in anxiety was greater in the experimental group than in the sham group, confirmed by both subjective (psychometric) measures and objective (biological) measures. This was demonstrated to be associated with the real neurofeedback, though a nonspecific (placebo) effect likely also contributed.

(c) 2020 Elsevier Masson SAS. All rights reserved.

\section{Introduction}

Electroencephalographic (EEG) neurofeedback (NF) is the oldest technique within the field of neuromodulation. In NF training, the participant learns to self-control his/her brain activity according to operant principles, with the aim of improving a variety of mental states, measured through behavioral or physiological variables, in clinical or nonclinical conditions [13]. Previous studies have shown the benefits of NF for attention deficit hyperactivity disorder (ADHD) patients trained to inhibit theta $(4-7 \mathrm{~Hz})$ activity while enhancing middle beta $(16-21 \mathrm{~Hz})$ rhythms [for a recent meta-analyses, see ref 29], especially for the impulsive hyperactive (ADHD-HI) or combined (ADHD-C) types of presentation [10]. In an effort to broaden the indications for NF, Gruzelier [11] highlighted the interest of investigating the putatively calming effect of such training, implicit in its clinical evidence for ADHD, with the aim of applying this tool to the therapeutic treatment of pathologies related to stress and anxiety. The author showed an increase in calmness only in relation to training sensorimotor rhythm (SMR; also known as low beta, $12-15 \mathrm{~Hz}$ ), as opposed to middle beta training in healthy participants. This helped direct interest in NF from the cognitive to the affective/mood field. The first reviews addressing the efficacy of EEG-NF for the treatment of depression and anxiety, including post-traumatic stress disorder (PTSD), reported encouraging results [12]. More recently, clinical guidelines from the Canadian Agency for Drugs and Technologies in Health (CADTH) [6], suggested that, compared with no treatment, there is a statistically significant symptomatic improvement when using NF in patients with PTSD or generalized anxiety disorder (GAD). Moreover, according to the Applied Psychophysiology and Biofeedback International Society, NF has reached at least a level of 4 in efficacy for anxiety [9]. Nevertheless, other guidelines and review reports are much more cautious, pointing out a scarcity of robust, methodologically valid evidence [19] and highlighting that, for mental disorders other than ADHD, research is too limited to warrant the use of EEG neurofeedback in clinical psychiatric practice [4].

One strategy for adding validity to the use of NF for mood disorders is to measure the effects of the training not only through subjective variables (i.e. with psychometric tests) but also through objective correlates (i.e. with biological variables). If we consider cortisol as a proven biomarker of stress, as exposed in recent reviews [2], then it is reasonable to expect some decrease of cortisol levels when implementing a clinical intervention which claims to promote calmness and relaxation. However, this type of report regarding NF is still scarce. One study [1] showed the benefits of NF training in a clinical case of anxiety by measuring both psychological symptomatology and salivary cortisol, to find that both diminished significantly with treatment. In another recent report, the perceived stress, clinical symptoms and serum levels of cortisol diminished in a sample of traumatic brain-injured patients treated with NF sessions [7]. Given this scenario, additional multilevel, psychophysiological explorations of NF training in healthy humans are needed to better understand its global effects and neural basis, before NF can be widely recommended in psychiatric practice.

As the literature shows the feasibility of a short-term approach with a variety of EEG protocols and studies [see a review in 24], we were interested in the possibility of obtaining mood changes after exposure to only one session of NF training. Thus, the primary endpoint of this study was to explore a putatively immediate calming effect on mood after a single session of $\uparrow S M R / \downarrow$ theta NF training in healthy people. We expected to observe both subjective changes of improvement through self-reporting (psychometric measures) and objective correlates in the form of hormonal (salivary cortisol) and neurophysiological (EEG frequency band power analysis) measures. In order to confirm such changes, we applied a pretest-posttest design and, according to recent recommendations for the research of NF in healthy adults [23], we included both an experimental group receiving real NF and a control group receiving a placebobased sham NF. Moreover, we followed criteria proposed by Zoefel et al. [32] for optimal research in NF studies, thus implying the following three points. First, we expected to obtain a good trainability, resulting in the desired EEG changes being limited to the trained EEG bands. Second, we expected to confirm EEG band independence, with lack of corresponding change in untrained bands. Third, we also expected to obtain a good interpretability, and thus to observe the improvement effects only in the experimental group.

\section{Methods}

\section{Participants}

A total of 32 right-handed young men, volunteer undergraduate students, between 18 and 28 years old (mean age $=21.81$, S.D. $=2.5$ ), were selected to take part in the experiment, and they received a pen-drive in gratitude for their participation. We choose male subjects because the rationale for this study emerged from the use of NF in patients with $A D H D$, of whom more than two thirds are male according to recent data [25]. The sample had no self-reported history of major depression or other psychiatric disorders, medical illness, chronic pharmacological treatment or drug consumption. None of them had previous knowledge or experience with NF training. All were 
instructed to abstain from eating, drinking (except water) and smoking for at least $1 \mathrm{~h}$ prior to the experiment. All participants signed the informed consent and were treated in accordance with "Ethical Principles of Psychologists and Code of Conduct" [3]. All procedures were in accordance with the ethical standards of the institutional research committee of the U.V. and with the 1964 Helsinki declaration and its later amendments.

\section{Psychometric measures of mood: POMS and STAI}

A Spanish adaptation [5] of the Profile of Mood State (POMS) questionnaire [18] and a Spanish adaptation [8] of the State Trait Anxiety Inventory (STAI) [27] were used to evaluate mood. Regarding the POMS, three dimensions of mood were evaluated, describing how the participant was feeling in that moment. The Tension/Anxiety scale was explored with the following adjectives: tense, agitated, about to burst, uncontrolled, relaxed, disturbed, restless, nervous and anxious. The Depression/Dejection scale was explored with the following adjectives: unhappy, hurt, sad, dejected, hopeless, clumsy, discouraged, lonely, miserable, depressed, desperate, helpless, useless, terrified and guilty. The Anger/Hostility scale was explored with the following adjectives: angry, enraged, irritable, resentful, annoying, spiteful, bitter, fighting, rebellious, disappointed, infuriated and ill-tempered. Regarding the STAI, we explored the anxiety state dimension by asking the participant how he was feeling in that moment according to the following phrases: "I feel calm", "I feel safe", "I am tense", "I am upset", "I feel comfortable", "I feel disturbed", "I am concerned about possible future misfortunes", "I feel rested", "I feel distressed", "I feel warm", "I have confidence in myself", "I feel nervous", "I'm unsettled", "I feel oppressed", "I'm relaxed", "I feel satisfied", "I'm worried", "I feel dazed and overexcited", "I feel cheerful", and "I feel good right now'. Both instruments are well-established, factor analytically derived measures of psychological distress, with high reliability and validity levels.

\section{Biological correlates of mood: cortisol measurements}

The participants provided four saliva samples, by depositing $5 \mathrm{~mL}$ of saliva in plastic vials during no more than $5 \mathrm{~min}$. The samples were centrifuged at $3000 \mathrm{rpm}$ for $15 \mathrm{~min}$, resulting in a clear supernatant with low viscosity that was stored at $-20 \mathrm{C}$ until the analyses were performed. The samples were analyzed by Salimetrics commercial salivary cortisol enzyme-linked immunosorbent assay kit (Newmarket, UK) in the Laboratory of Social Cognitive Neuroscience of the University of Valencia (Spain). The sensitivity of the assay was $0.007 \mathrm{ug} / \mathrm{dL}$. All samples were measured in duplicate and in the same trial. The within- and inter-assay variation coefficients were all below $10 \%$.

\section{Neurophysiological correlates of mood: EEG recording and neurofeedback}

EEG signals were recorded, processed, filtered and represented using the BioGraph Infiniti EEG Suite SA7950 Software (which amplifies the amplitude of the frequency bands), and the hardware Pro Comp 2 Infinity (Thought Technology Ltd; Montreal, Quebec) for sending the signal to the computer. The EEG was used for both recording and feedback, which was sampled at $256 \mathrm{~Hz}$ samples/second and sent to the computer by the A/D converter. The potential field was recorded through a monopolar electrode connection. In accordance with the International 10-20 system, an active scalp electrode was placed at $\mathrm{Cz}$ with the reference and ground electrode on the right and left earlobe, respectively. Impedance was maintained below $10 \mathrm{~K} \Omega$ and artefact rejection thresholds were set to suspend feedback when eye movements or other motor activity would cause EEG fluctuations. The ongoing EEG at site $\mathrm{Cz}$, was analysed by fast Fourier transform (FFT), band-pass filtered (from 0.1 to $60 \mathrm{~Hz}$ ) and notch filtered $(50 \mathrm{~Hz})$, in order to eliminate electrical interference and continuously measure the amplitude of the measured bands.

Resting EEG was analysed in periods of 5 minutes, one period before and another period after the NF training. In these periods, 3 bands were analyzed: theta $(4-7 \mathrm{~Hz})$, SMR $(12-15 \mathrm{~Hz})$, and middle beta $(16-21 \mathrm{~Hz})$. We thus obtained baseline evaluation of these three bands for comparison with the post training condition. SMR and theta band activities were also important to determine the initial threshold for the subsequent reinforcement in the NF training. Regarding the middle beta band, it was included in the analyses to discard its influence on mood, according to the hypothesis being tested and also following the proposal by Gruzelier [11]. Pre-post training amplitudes were evaluated and compared for theta, SMR and middle beta, but only theta and SMR were trained in the online NF session (explained below). Other bands (delta, alpha, high beta) were not included in the analyses since this was beyond the aim of the study. PreNF and post-NF recording was performed with eyes opened and in a resting state; that is, participants did not perform any type of activity that required a cognitive load and did not receive feedback while recording their brain activity. The only instruction the participants received was: "just relax and look at the symbol (a cross) that will appear on the screen of the computer during the next 5 minutes"'.

The 5-min EEG baseline and post-NF session recordings were completed for all participants.

\section{Neurofeedback}

For the NF training session, EEG amplitude was measured online for two bands: theta $(4-7 \mathrm{~Hz})$ and SMR $(12-15 \mathrm{~Hz})$, in microvolts, $\mu \mathrm{V}$, and peak-to-peak. During the online session of NF, feedback thresholds were automatically reset between blocks to maintain a constantly increasing level of reinforcement throughout the training session. When we talk about "block" within the NF training we are referring to the way in which the software collects data related to the activity being recorded in order to give (or not) the corresponding reward. Specifically, each block lasts $1 \mathrm{sec}$ ond and, in each second, four means of each frequency are collected. If the participant manages to comply with the threshold marked in $3 / 4$ of the means obtained, the reward is given and, for the next block (the next second), the threshold will be readjusted in 0.10 microvolts. Note that the present training protocol involved two bands with different 
targets (to enhance SMR and to inhibit theta); therefore, the threshold marked for the SMR band was to reach $3 / 4$ means above the mean amplitude for the previous block, and for the theta band to reach $3 / 4$ means below the mean amplitude for the previous block. Therefore, the participant must meet both thresholds in order to continuously obtain the reward. The session provided 1800 blocks without stopping, thus 30 minutes of NF training. An audio-visual thresholddependent protocol was used as reward stimuli feedback. The reward was presented on the computer screen and was selected from the options included in the BioGraph Infiniti software. It was a cyclically-repeated, changing animation of a landscape, accompanied by instrumental and neutrally valenced music (without human voice). If the participant accomplished the predefined thresholds for both bands, he could see the animation and listen to the music without interruptions. If the participant could not adjust to the thresholds, the audio would stop, and the image would not change. The only instruction the participants received was: "relax, listen to the music and watch the computer screen, with the intention of keeping the music going and the image moving throughout the duration of the training'.

The procedure detailed above for the online NF training (real NF) was only implemented in the experimental group. The control group underwent a condition of sham NF. In this condition all procedures followed the same steps regarding electrode placement, 5-min signal recordings, and instructions given, but the type of online EEG signal used to give the feedback was different: in the sham condition the participant received on the screen a previously recorded EEG signal of another participant as a not-contingent reward. The sham NF therefore represents a fake NF condition, over which the participant has no real control (conscious or not) despite his attempts to carry out the instructions.

\section{Design and procedure}

Participants were randomly allocated to a session with real NF training (16 men) or with sham NF training (16 men), with similar age scores. They were blind to group assignment and to the existence of a sham NF condition. All participants provided informed written consent. For the test, they were seated in a comfortable chair about $0.5 \mathrm{~m}$ from the monitor. We collected four measures of cortisol (C) and two measures of mood according to the design C-MOODC-NF-C-MOOD-C. Participants in the experimental condition received 30 minutes of real feedback. They were not provided with any specific instructions about how to achieve the task except for the general instruction commented in the above section, and just were encouraged to relax, in line with literature recommendations [31]. The instructions given in the sham condition were the same but these participants received 30 minutes of not-contingent feedback.

\section{Data analyses}

Firstly, we explored the differences between pre- and postNF measures in each group (using only the pre- and post-NF measure for cortisol). After testing for normality, we applied either a Student's $t$-test for related variables or a Wilcoxonrank test to raw scores, with size effects estimations by
Cohen's d (G*Power formulae). Additionally, and given that we had four measures for cortisol, we calculated an area under the curve with respect to the increase (AUCi) according the formula detailed in [21]. The AUCi is routinely used in endocrine research to detect possible associations between repeated measures and other variables, to incorporate multiple time points. Following this, and in order to compare the magnitude of the change after the NF between the experimental and the control group, we performed subtractions, according to the formulae $=$ [post-NF minus pre-NF] +100 . We tested the normality on the transformed variables to apply either a Student's $t$-test for independent samples or a Mann-Whitney $U$ test, with size effect estimations by Cohen's $d$ and including a power calculation for the significant comparisons. Finally, Spearman correlations for each group separately and with Bonferroni corrections were applied, to test the relationships regarding magnitude of change. All analyses were performed using the SPSS 24 statistical package set. Data are presented as means and standard deviations (SD).

\section{Results}

\section{Analyses of raw scores for each group (Table 1, left)}

Regarding psychometric measures of mood results, in the experimental group we observed a significant decrease for POMS-tension-anxiety [z $(15)=-1.93 ; P<0.05 ; d=0.42$; power $=0.5]$ and for POMS-anger-hostility $[t \quad(15)=2.45$; $P<0.02 ; d=0.60$; power $=0.7]$ after the real NF. The control group also showed a significant decrease in POMS-angerhostility scores $[t(15)=2.65, P<0.01 ; d=0.63$; power $=0.7]$ but interestingly showed a significant increase in STAI State Anxiety Scores [ $t(15)=-3.47 ; P<0.003 ; d=0.90$; power $=0.9]$ after the sham NF.

Regarding biological correlates, we observed a significant decrease of cortisol levels both for the experimental [ $t(15)=4.81 ; P<0.001 ; d=0.85 ;$ power $=0.9]$ and for the control $[t(15)=6.60 ; P<0.001 ; d=0.90$; power $=0.9]$ groups after the task.

Regarding the neurophysiological correlates through EEG results, the experimental group showed a significant increase in SMR band amplitude $[t(15)=3.43, P<0.004$; $d=0.88$; power $=0.9]$ and also in the middle beta band amplitude $[t(15)=2.33, P<0.03 ; d=0.58$; power $=0.7]$ after the real NF, with no significant changes for theta band. The control group performed without significant differences after the sham NF for any of the three EEG bands.

\section{Group comparisons in magnitude of change (Table 1, right)}

Regarding psychometric measures of mood results, the experimental group significantly diminished their POMSanxiety score $[F(1,30)=7.49 ; P<0.01 ; d=0.97$; power $=0.8]$ compared to the control group.

Regarding biological correlates, the experimental group showed a greater but non-significant decrease in cortisol compared to the control group $[t(30)=-1.89 ; P=0.07$; 
Table 1 Mean (standard deviation) regarding the relevant variables pre- and post-real neurofeedback (NF) for the experimental group or sham neurofeedback (NF) for the control group, with level of significance ( $P$-values) and size effect (Cohen's $d$ ). Right: mean (standard deviation) of variables representing magnitude of change [subtractions (Sub) and AUCi] for each group separately.

\begin{tabular}{|c|c|c|c|c|c|c|c|c|c|c|c|}
\hline Real NF & Pre-NF & Post-NF & $p ; d$ & Sham NF & Pre-NF & Post-NF & $p ; d$ & $\%$ change & Real NF & Sham NF & $p ; d$ \\
\hline SMR & $2.70(0.34)$ & $2.94(0.44)$ & $0.004 ; 0.88$ & SMR & $3.72(0.84)$ & $3.75(0.90)$ & n.s. & SubSMR & $100.23(0.27)$ & $100.02(0.30)$ & $0.04 ; 0.73$ \\
\hline Z & $7.48(1.64)$ & $7.52(2.05)$ & n.s. & Z & $7.50(1.30)$ & $7.64(1.61)$ & n.s. & SubZ & $100.03(2.22)$ & $100.13(1.32)$ & n.s. \\
\hline B & $3.87(0.38)$ & $4.57(1.34)$ & $0.03 ; 0.58$ & B & $5.33(1.08)$ & $5.32(0.96)$ & n.s. & SubB & $100.70(1.19)$ & $99.98(0.40)$ & n.s. \\
\hline STAI State & $12.06(6.9)$ & $14.56(8.49)$ & n.s. & STAI state & $10.25(5.11)$ & $16.81(8.39)$ & $0.003 ; 0.90$ & SubSTAI state & $102.50(5.89)$ & $106.56(7.54)$ & n.s. \\
\hline POMS ANX & $8.18(5.33)$ & $6.31(3.26)$ & $0.05 ; 0.42$ & POMS ANX & $7.62(2.65)$ & $8.87(3.50)$ & 0.07 & SubPOMS ANX & $98.12(3.77)$ & $101.25(2.56)$ & $0.01 ; 0.97$ \\
\hline POMS DEP & $2.75(2.08)$ & $2.81(3.41)$ & n.s. & POMS DEP & $8.37(7.13)$ & $7.62(5.77)$ & n.s. & SUbPOMS DEP & $100.06(2.81)$ & $99.25(2.84)$ & n.s. \\
\hline POMS ANG & $6.81(3.56)$ & $5.00(3.77)$ & $0.02 ; 0.60$ & POMS ANG & $10.18(8.82)$ & $8.12(6.38)$ & $0.01 ; 0.63$ & SubPOMS ANG & $98.18(2.94)$ & $97.93(3.10)$ & n.s. \\
\hline $\mathrm{C}$ & $4.29(2.6)$ & 2.53 (1.35). & $0.001 ; 0.85$ & C & $2.98(1.17)$ & $1.97(0.74)$ & $0.001 ; 0.90$ & $\begin{array}{l}\text { SubC; } \\
\text { AUCi }\end{array}$ & $\begin{array}{l}98.24(1.46) ; \\
-239.31(194.91)\end{array}$ & $\begin{array}{l}98.99(0.61) ; \\
-123.37(94.76)\end{array}$ & $\begin{array}{l}\text { n.s.; } \\
0.04 ; 0.75\end{array}$ \\
\hline
\end{tabular}

SMR: sensorimotor rhythm; Z: theta; B: beta; STAI: State Trait Anxiety Inventory; POMS: Profile of Mood State; ANX: anxiety; DEP: depression; ANG: anger; C: cortisol; AUCi: area under the curve regarding the increment; n.s.: not significant. 
$d=0.67]$. However, when we considered the four measures of cortisol that we had taken into the composed AUCi score, the experimental group had a significantly greater decrease of their AUCi $[t(30)=-2.13 ; P<0.04 ; d=0.75$; power $=0.6]$ compared to the control group.

Regarding EEG results, the experimental group showed a significantly greater increase in SMR amplitude $[t(30)=2.05$; $P<0.04 ; d=0.73$; power $=0.6]$ compared to the control group.

On the other hand, regarding the correlations among magnitudes of change for each group separately, the only correlation that reached statistical significance after a Bonferroni correction was the positive correlation between cortisol and POMS-anger-hostility that was observed in the experimental group [rho $(16)=0.71 ; P<0.002]$. We did not observe any significant correlation between the EEG bands and mood or cortisol scores in any of the two groups.

\section{Discussion}

We aimed at testing the effectivity of one single session of $\uparrow S M R / \downarrow$ theta NF training for mood modulation in healthy men, taking into account trainability, independence, and interpretability of the results [32], including a sham NF group to assure these goals. We expected both subjective changes of improvement as measured through self-reporting and objective changes of improvement through hormonal correlates (cortisol) and neurophysiological correlates (EEG frequency band power analysis).

Regarding subjective self-reporting, the participants receiving real NF showed reduced feelings of tension/anxiety. The participants under the sham NF training showed an opposite pattern for anxiety: the fact of performing fake NF training increased their anxiety levels. On the other hand, both groups reported feeling less anger/hostility after the task (real or fake NF). We then looked for biological markers of improvement using salivary cortisol [2]. We found that all participants showed significant decrease of cortisol after the training, whether they performed real or sham NF. However, closer inspection of the data through the exploration of the magnitude of change through the AUCi revealed a significantly higher effect (greater decrease of cortisol) for participants who received real NF. Finally, from a neurophysiological perspective, the group receiving sham NF showed no significant EEG changes, in contrast with the experimental group, which showed signs of enhanced SMR band after training with real NF, in line with other reports $[17,30]$. Taken as a whole point, these results point to a combination of specific (true) and nonspecific (placebo) effects of NF training. Among the nonspecific factors [22] most likely to have affected the present study, the technological interface and the characteristics of the instructions (to stay relaxed) may have affected the sham NF group and resulted in a certain mood improvement.

On the other hand, as commented above, we were interested in testing the criteria of Zoefel et al. [32] with regards to optimal research in NF. To assess trainability and independence, we expected that a significant change in SMR and theta bands along with no changes for the middle beta band, would appear only in the experimental group after the real NF. Such assumption was confirmed in the case of the SMR band, but on the other hand, the real NF had no apparent effects in the theta band. This observation was in contrast with other reports which have claimed theta as the most trainable band, at least in children with ADHD, who have been reported to show an excess of theta activity [26]. It must be noted that healthy people do not usually have such an imbalance of theta, but our design included its manipulation in the EEG protocol given that impulsive and emotionally-driven behaviors have also been associated with an excess of theta activity [15]. In the light of the results, we advise that future studies should aim to train only the SMR band if the goal is mood modulation. We agree with the suggestion of Rogala et al. [23] that the use of protocols with two bands to train may be more difficult for the participants to perform. Thus, in our study the trainability criterion was accomplished by SMR but not by theta. Regarding the independence criterion, we observed that the untrained middle beta band increased after the real NF, a demonstration that the training of SMR for up-regulation was accompanied by changes in the flanking frequency band, which challenges the idea of frequency-specific EEG-NF protocols and points to their interdependence. The issue of the specificity (or not) of the NF training to a certain band has been questioned in various recent reports $[14,16]$ since it is common to find such parallel changes in untrained bands. For instance, Kober et al. [17] reported that SMR-based NF training led to a linear increase in the high beta band $(21-35 \mathrm{~Hz})$ similar to our findings. Moreover, regarding SMR/beta relations, it has been suggested that the increase in middle or high beta activity during SMR NF training may result merely from the compliance with the general instructions to avoid movement [20]. To sum up, the EEG protocol applied in this study showed mixed results regarding EEG band trainability and also a lack of independence between bands. Regarding the interpretability of the data (third criterion of Zoefel et al., [32]), this was only partially accomplished, since we observed some measures of improved mood with sham NF, and we could not demonstrate from the correlational analyses an unequivocal connection between the observed EEG modulation and the psychophysiological results.

The main goal of our study was to assess NF as a technique with real capability of neuromodulation to improve mood, beyond any placebo effect. The literature has criticized the scarcity of robust evidence precluding conclusions in this regard [28] and strongly encourages the implementation of a sham NF condition as we did [23]. In the present study, performed with an appropriate design, we observed a clear capacity of real NF to modulate, train, and change EEG outcomes, but the feelings of anger and the cortisol levels of both groups were similarly better after the experiment, irrespective of whether sham or real NF was used. However, we also noticed significantly more pronounced effects in the desired direction after the real NF and an opposite effect on anxiety feelings, which decreased after the real but increased after the sham NF. As a whole, our results were similar to those observed by Gruzelier [11] and seemed to partially confirm his hypothesis about an association between calm feeling and SMR. This hypothesis posits that SMR activity is associated with "internal inhibition" due to attenuation of somatosensory information to the cortex during SMR activity, which could lead to a calm feeling. Our results partially support this approach, 
extending the data to cortisol, a physiological marker of stress response.

\section{Conclusion}

Mood modulation seemed to be partially due to real NF, though evidence for nonspecific factors affecting the task was also obtained. Given that the best effects were observed for the anxiety dimension, it would be interesting to replicate this study with broader samples of both genders and also with clinical samples suffering from mood disorders involving anxiety.

\section{Disclosure of interest}

The authors declare that they have no competing interest.

\section{Acknowledgements}

This study was supported by the Spanish Ministry of Education (grant number FPU-AP2012-05836).

\section{References}

[1] Aliño M, Gadea M, Hidalgo V, Pérez V, Sanjuán J. An effective neurofeedback training, with cortisol correlates, in a clinical case of anxiety. Univ Psychol 2016;15:1-10.

[2] Allen AP, Kennedy PJ, Cryan JF, Dinan TG, Clarke G. Biological and psychological markers of stress in humans: focus on the Trier Social Stress Test. Neurosci Biobehav Rev 2014;38:94-124.

[3] American Psychological Association. Ethical principles of psychologists and code of conduct. Am Psychol 1992;47:1597-611.

[4] Arns M, Batail JM, Bioulac S, Congedo M, Daudet C, Drapier D, et al. Neurofeedback: one of today's techniques in psychiatry? Encephale 2017;43:135-45.

[5] Balaguer I, Fuentes I, Meliá JL, García-Merita ML, Perez-Regio G. El perfil de los estados de ánimo (POMS): baremo para estudiantes valencianos. J Sport Psychol (RPD) 1993;4:39-52.

[6] Banerjee S, Argáez C. Neurofeedback and biofeedback for mood and anxiety disorders: a review of clinical effectiveness and guidelines. Ottawa (ON): CADTH; 2017.

[7] Bennet CN, Gupta RK, Prabhakar P, Christopher R, Sampath S, Thennarasu K, et al. Clinical and biochemical outcomes following EEG neurofeedback training in traumatic brain injury in the context of spontaneous recovery. Clin EEG Neurosci 2018;49:433-40.

[8] Buela-Casal G, Guillén-Riquelme A, Seisdedos Cubero N. Cuestionario de ansiedad estado-rasgo 8th ed. [Inventory of state-trait anxiety, 8th ed]. Madrid: TEA ediciones; 2011.

[9] Carrobles JA. Bio/neurofeedback. Clin Salud 2016;27:125-31.

[10] Cueli M, Rodríguez C, Cabaleiro P, García T, González-Castro P. Differential efficacy of neurofeedback in children with ADHD presentations. J Clin Med 2019;8:204.

[11] Gruzelier JH. Differential effects on mood of 12-15 (SMR) and 15-18 (beta1) Hz neurofeedback. Int J Psychophysiol 2014;93:112-5.

[12] Hammond DC. Neurofeedback with anxiety and affective disorders. Child Adolesc Psychiatr Clin N Am 2005;14:105-23.

[13] Herrmann CS, Strüber D, Helfrich RF, Engel AK. EEG oscillations: from correlation to causality. Int J Psychophysiol 2016;103:12-21.

[14] Jurewicz K, Paluch K, Kublik E, Rogala J, Mikicin M, Wróbel A. EEG neurofeedback training of beta band $(12-22 \mathrm{~Hz})$ affects alpha and beta frequencies - A controlled study of a healthy population. Neuropsychologia 2018;108:13-24.

[15] Knyazev GG. Motivation, emotion, and their inhibitory control mirrored in brain oscillations. Neurosci Behav Rev 2007;31:377-95.

[16] Kober SE, Witte M, Neuper C, Wood G. Specific or nonspecific? Evaluation of band, baseline, and cognitive specificity of sensorimotor rhythm and gamma-based neurofeedback. Int J Psychophysiol 2017; 120:1-13.

[17] Kober SE, Witte M, Stangl M, Valjamae A, Neuper C, Wood G. Shutting down sensorimotor interference unblocks the networks for stimulus processing: an SMR neurofeedback training study. Clin Neurophysiol 2015;126:82-95.

[18] McNair DM, Lorr M, Droppleman LF. Profile of mood states manual. San Diego, CA, USA: Educational and Industrial Testing Services; 1992.

[19] Micoulaud-Franchi JA, McGonigal A, Lopez R, Daudet C, Kotwas I, Bartolomei F. Electroencephalographic neurofeedback: level of evidence in mental and brain disorders and suggestions for good clinical practice. Neurophysiol Clin 2015;45:423-33.

[20] Pimienta MG, van Run C, de Fockert JW, Gruzelier JH. Neurofeedback of SMR and Beta1 frequencies: an investigation of learning indices and frequency-specific effects. Neuroscience 2018;378:211-24.

[21] Pruessner JC, Kirschbaum C, Meinlschmid G, Hellhammer $\mathrm{DH}$. Two formulas for computation of the area under the curve represent measures of total hormone concentration versus time-dependent change. Psychoneuroendocrinology 2003;28:916-31.

[22] Raz A, Michels R. Contextualizing specificity: specific and nonspecific effects of treatment. Am J Clin Hypnosis 2007; 50:177-82.

[23] Rogala J, Jurewicz K, Paluch K, Kublik E, Cetnarski R, Wróbel A. The do's and don'ts of neurofeedback training: a review of the controlled studies using healthy adults. Front Hum Neurosci 2016;10:301.

[24] Ros TBBJ, Lanius RA, Vuilleumier P. Tuning pathological brain oscillations with neurofeedback: a systems neuroscience framework. Front Hum Neurosci 2014;8:1-22.

[25] Rowland AS, Skipper BJ, Umbach DM, Rabiner DL, Campbell RA, Naftel AJ, et al. The prevalence of ADHD in a population-based sample. J Attent Dis 2015;19:741-54.

[26] Sitaram R, Ros T, Stoeckel L, Haller S, Scharnowski F, LewisPeacock $J$, et al. Closed-loop brain training: the science of neurofeedback. Nat Rev Neurosci 2017;18:86-100.

[27] Spielberger CD, Gorsuch R, Lushene R. Manual for the State Trait Anxiety Inventory. Palo Alto: Consulting Psychologist Press; 1970.

[28] Thibault RT, Lifshitz M, Raz A. The self-regulating brain and neurofeedback: experimental science and clinical promise. Cortex 2016;74:247-61.

[29] Van Doren J, Arns M, Heinrich H, Vollebregt MA, Strehl U, Loo KS. Sustained effects of neurofeedback in ADHD: a systematic review and meta-analysis. Eur Child Adolesc Psychiatry 2019;28:293-5.

[30] Weber E, Köberl A, Frank S, Doppelmayr M. Predicting successful learning of SMR neurofeedback in healthy participants: methodological considerations. Appl Psychophysiol Biofeedback 2011;36:37-45.

[31] Witte M, Kober SE, Ninaus M, Neuper C, Wood G. Control beliefs can predict the ability to up-regulate sensorimotor rhythm during neurofeedback training. Front Hum Neurosci 2013;7:478.

[32] Zoefel B, Huster RJ, Herrmann CS. Neurofeedback training of the upper alpha frequency band in EEG improves cognitive performance. Neuroimage 2011;54:1427-31. 